\title{
Dynamics of a single vortex line in a condensate
}

\author{
V. Bretin, P. Rosenbusch, and J. Dalibard \\ Laboratoire Kastler Brossel* ${ }^{*} 24$ rue Lhomond, 75005 Paris, France
}

\begin{abstract}
We study experimentally the line of a single vortex in a rotating prolate Bose-Einstein condensate confined in a harmonic potential. In agreement with predictions, we find that the vortex line is in most cases curved at the ends. We also present measurements of the quadrupole oscillation of the condensate in presence of a single vortex. A theoretical treatment to account for the short time and long time behaviors of these modes is developed. Finally, we use these measurements to infer the angular momentum per particle and relate it to the shape of the vortex line.
\end{abstract}

\section{Introduction}

The rotation of a macroscopic quantum object is a source of spectacular behaviors. A fascinating phenomenon is the nucleation of vortices under rotation. In superfluid liquid helium contained in a cylindrical bucket rotating around its axis $z$, one observes the nucleation of quantized vortices for a sufficiently large rotation frequency $\Omega$ [1]. A similar phenomenon occurs in Bose-Einstein condensates confined in a rotating harmonic trap [2, 3, 4, 5, 6]. These quantized vortices in superfluids are analogous to the quantized flux lines in superconductors [7]. Among the remaining problems, is the shape of a vortex/flux line and the study of its time evolution. The observation of inclined flux lines in superconductors was only possible due to recent advances in electron microscopy 8. In gaseous condensates, one has comparably easy access to the vortex line because the density of the atom cloud is low. A few disordered vortex lines have been observed by taking tomographic images perpendicular to the long axis of a cigar shaped condensate 4. An array of more than 30 vortex lines in a pancake shaped condensate has been observed by transverse imaging of the whole atom cloud $[9]$.

In this article we report the full length observation of a single vortex line in a cigar shaped condensate. We find that as a result of spontaneous symmetry breaking the line is generally bent [10]. Our experimental results confirm recent predictions, in which the shape of the vortex line minimizing the energy of the gas was derived for a given rotation frequency [11, 12, 13, 14, 15.

We also present measurements of the angular momentum of the rotating condensate, based upon surface wave spectroscopy. We develop the theoretical framework to account for the excitation of the quadrupole surface modes of the condensate. We then relate the measured angular momentum of the gas to the bending of the vortex line and its deviation from the center. 


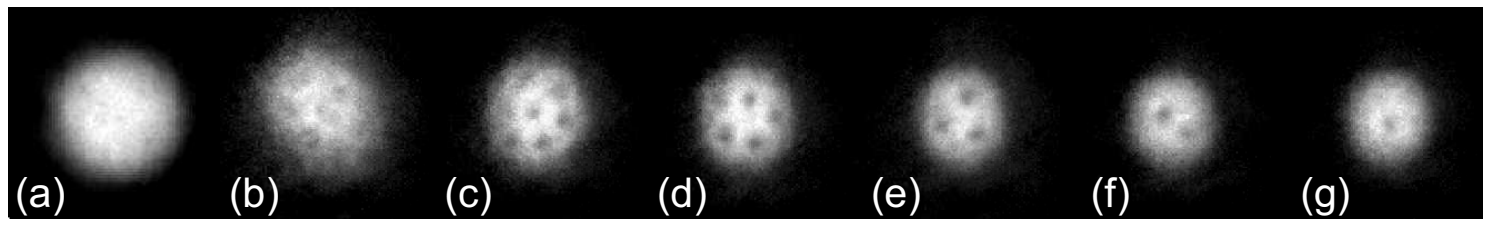

Figure 1. Image of the condensate with the probe beam propagating along the $z$ axis.

a) Before stirring. b) Just after stirring $(\tau=0)$. c) $\tau=150 \mathrm{~ms}$. d) $\tau=500 \mathrm{~ms}$. e) $\tau=800 \mathrm{~ms}$. f) $\tau=1200 \mathrm{~ms}$. g) $\tau=1800 \mathrm{~ms}$.

\section{Preparation of a single vortex}

\subsection{Experimental setup}

Our ${ }^{87} \mathrm{Rb}$ condensate is formed by radio-frequency (rf) evaporation of $10^{9}$ magnetically trapped atoms in the $F=m_{F}=2$ state. The longitudinal and transverse frequencies of the trap are respectively $\omega_{z} / 2 \pi=11.8 \mathrm{~Hz}$ and $\left(\omega_{x}+\omega_{y}\right) / 4 \pi=\omega_{\perp} / 2 \pi \sim 100 \mathrm{~Hz}$ (the $x$ axis is vertical). Because gravity slightly displaces the center of the trap with respect to the magnetic field minimum, the potential in the $x y$ plane is not perfectly isotropic and we measure a $1 \%$ relative difference between $\omega_{x}$ and $\omega_{y}$.

The condensation threshold is reached at $T_{\mathrm{c}} \sim 300 \mathrm{nK}$, with $N_{\mathrm{c}} \sim 2 \times 10^{6}$ atoms. We cool to typically $T \sim 90 \mathrm{nK}$ which corresponds to a condensate with $N_{0} \sim 5 \times 10^{5}$ atoms and a chemical potential $\mu \sim 70 \mathrm{nK}$. It is obtained using $\nu_{\mathrm{f}}=\nu_{0}+10 \mathrm{kHz}$ as the final rf, where $\nu_{0}$ is the frequency at which the trap is emptied. During the rest of the experimental cycle, we maintain the evaporation rf at an adjustable level, typically $\nu=\nu_{0}+12 \mathrm{kHz}$, to control the temperature.

\subsection{Vortex nucleation}

Once the condensate is formed, we use an off-resonant laser beam to impose on the trapping potential an elliptic anisotropy in the $x y$ plane 3 . The wavelength of the beam is $852 \mathrm{~nm}$, its power $0.1 \mathrm{~mW}$ and its waist $20 \mu \mathrm{m}$. Acousto-optic modulators deflect the position of the beam in the $x y$ plane, thereby rotating the potential anisotropy at a frequency of $\Omega / 2 \pi=70 \mathrm{~Hz}$. The potential created by the stirrer can be written :

$$
U(\mathbf{r})=\frac{\epsilon}{2} M \omega_{\perp}^{2}\left(X^{2}-Y^{2}\right),
$$

where the axes $X, Y$ are deduced from the fixed $x, y$ axes by a rotation by an angle $\Omega t$. $M$ is the atomic mass and the coefficient $\epsilon$ measures the relative strength of the stirring and the magnetic potential. We use typically $\epsilon \sim 4 \%$.

We apply this "laser stirrer" for $300 \mathrm{~ms}$, during which $\sim 7$ vortices are nucleated 16. The condensate then evolves freely in the magnetic trap for an adjustable time $\tau$. The atom distribution at $\tau$ is probed destructively by switching off the magnetic trap, letting the cloud expand during $t_{\mathrm{TOF}}=25 \mathrm{~ms}$ and performing absorption imaging.

The preparation of a single vortex line takes advantage of the slight static anisotropy of our magnetic trap, so that the angular momentum is not exactly a constant of 
motion. In a time $\tau \sim 1-2 \mathrm{~s}$, we observe a transition from a multi-vortex condensate to a condensate with a single vortex (see Fig. 1). This relatively long time levels the fluctuations that may occur during the nucleation process. Thereby we are able to reproduce a condensate with a single vortex on every experimental cycle. This vortex line can then be studied for a time $\tau \leq 10 \mathrm{~s}$.

In order to obtain an image of the full vortex line, we send two imaging beams aligned along the $y$ and $z$ directions, which probe the atom cloud simultaneously (Fig. 2a). The beams are combined onto a camera with the same magnification. During the expansion, the transverse dimensions $x$ and $y$ of the condensate are magnified by $\omega_{\perp} t_{\mathrm{TOF}} \sim 15$, while the longitudinal dimension is nearly unchanged [17]. It has been shown theoretically that the presence of a single vortex line does not alter this expansion and that the coordinates of the line are scaled by the same factors [14.

The transverse images in Fig. 20,c respectively obtained for $\tau=7.5 \mathrm{~s}$ and $\tau=5 \mathrm{~s}$ show the vortex line as a line of lower atomic density. Clearly this vortex line is not straight. It rather has the shape of a wide "U" or an unfolded " $\mathrm{N}$ ". The fraction of $\mathrm{N}$ vs. U shaped vortices depends on temperature. Mostly U's are observed at the lowest temperature $(T=75 \mathrm{nK})$, while $\sim$ half of the vortices are $N$-shaped for $T \geq 100 \mathrm{nK}$. Fig. $2 \mathrm{~d}$ has been obtained with a 7 -vortex lattice (one vortex at the center and the six other ones at the summits of an hexagon, such as in Fig. 11.). We have selected an image for which the lattice planes are parallel with the transverse imaging beam. The dark trace passing through the center of the condensate is the sum of 3 vortex lines. At the top and bottom, 2 lines lie on top of each other.
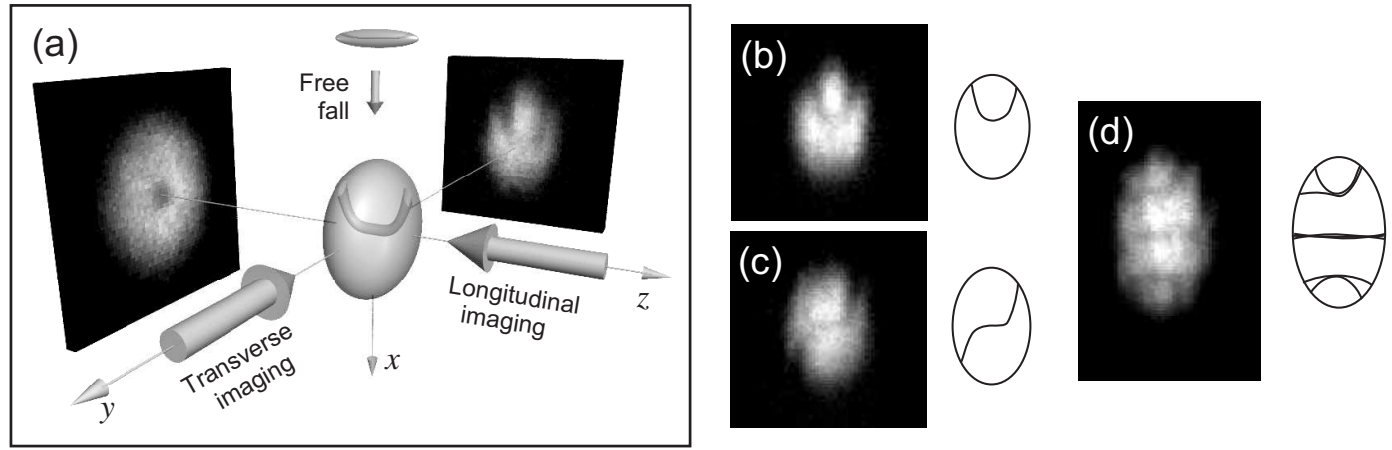

Figure 2. (a) Schematic of the imaging system allowing for an observation of the full vortex line. The initially cigar shaped condensate is imaged after $25 \mathrm{~ms}$ of time of flight leading to the inversion of the ellipticity in the $x z$ plane. Two beams image the atom cloud simultaneously along the longitudinal $(z)$ and transverse $(y)$ directions of the initial cigar. (b), (c) Transverse images of a condensate with a single vortex line respectively with a "U" and "N" shape. The schematic represents the form of the vortex line. (d) Transverse image of a condensate with 7 vortices. 

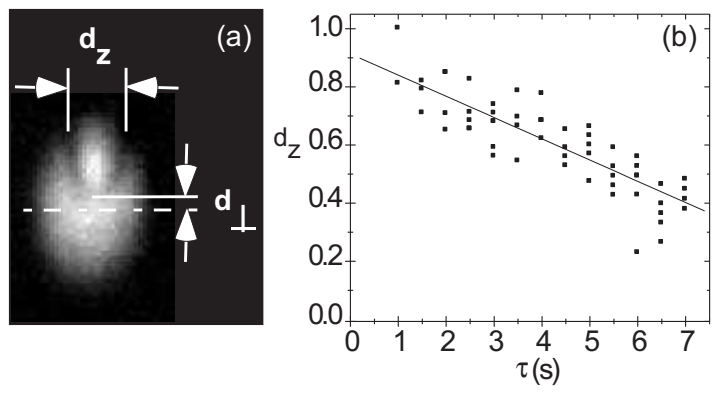

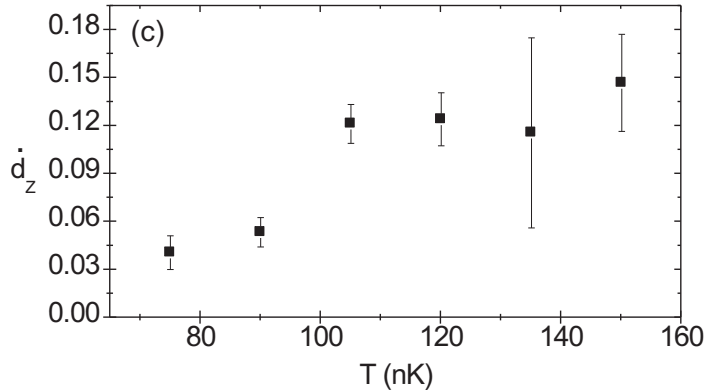

Figure 3. (a) Schematic of the extraction of $d_{z}$ and $d_{\perp}$ from the vortex line. (b) Evolution of $d_{z}$ with $\tau$. Each point corresponds to a single image. (c) Variation of $\dot{d}_{z}$ with the temperature $T$.

\subsection{Evolution of a single vortex}

In order to give a quantitative analysis of the time evolution of a condensate with a single vortex, we measure the distance along the $z$ direction between the two points where the vortex line leaves the condensate (Fig. [3a). Note that the ends of the vortex line in the transverse image are nearly always well visible with good contrast $(\sim 30 \%)$, even if the contrast of the line at the center of the condensate is poor (below 20\%). Therefore this measurement can be performed on most images. Normalization by the length of the condensate along $z$ leads to the quantity $d_{z}$.

In Fig. [ $3 \mathrm{~b}$, we plot $d_{z}$ as a function of $\tau$. Each point in the plot corresponds to a single image. The normalized length of the vortex line $d_{z}$ decreases quasi-linearly with time. The graph shows that after $\tau=7 \mathrm{~s}$ the vortex is still present (in $7 \mathrm{~s}$ the number of atoms is divided by a factor 2.5). This long lifetime is reminiscent of the MIT result [4, where a vortex array with more than 100 vortices was produced at $\tau=0$. The number of vortices was divided by 4 in $\sim 5 \mathrm{~s}$; however a single vortex could still be detected after $\tau=40 \mathrm{~s}$. In both experiments, it is clear that the decay time of the last vortex is much longer than the one of the initial array.

We have repeated this experiment for different temperatures $T$ during the free evolution time $\tau$. Fig. 3. displays the measured slopes as a function of temperature. We observe a quasi-linear decrease of $d_{z}$ for all temperatures. Our results can be compared to the one reported in [18, where the decay rate of an array of $\sim 100$ vortices as a function of temperature was measured. In [18, a $60 \%$ increase in temperature leads to an increase by a factor 17 in the decay rate. This variation is more dramatic than ours, indicating different decay mechanisms for a single vortex and for a large vortex array. In the latter case, the friction between the vortices and the thermal cloud makes the two components of the system stick together [19]. In the case of a single vortex, the most probable scenario is that the rotation of the thermal component rapidly stops due the residual static trap anisotropy [20]. The vortex line is then dragged to the edge of the condensate due to the friction between the two components [21]. 


\section{Angular momentum of a condensate with a single vortex}

An important parameter for the characterization of a rotating condensate is the average angular momentum per particle $L_{z}$. This quantity is related to the transverse quadrupole frequencies of the condensate $\omega_{ \pm}$by the formula :

$$
L_{z}=\frac{1}{2} M r_{\perp}^{2}\left(\omega_{+}-\omega_{-}\right)
$$

where $r_{\perp}^{2}$ stands for the average value of $x^{2}+y^{2}$ for the condensate. The physical basis of (2) is clear. In absence of angular momentum along the $z$ axis, the system is rotationally invariant and the two transverse quadrupole modes $m=+2$ and $m=-2$ have the same frequencies $\omega_{+}=\omega_{-}$(and also the same damping rates $\gamma_{+}=\gamma_{-}$). In presence of a vortex the symmetry between the modes $m= \pm 2$ is broken and (2) relates the corresponding lift of degeneracy $\omega_{+}-\omega_{-}$to $L_{z}$.

The result (2) was established in [22] for a rotating condensate with an arbitrary number of vortices in the Thomas Fermi limit. For a condensate with a well centered vortex it had been obtained in [23, 24, 25]. In this section we discuss the measurement of $\omega_{ \pm}$based on a percussional excitation of the condensate and we present the relation between the results of our measurements of $L_{z}$ and the shape of the vortex line.

\subsection{Measurement of $\omega_{ \pm}$from the precession of the condensate axes}

We first prepare the condensate with a single vortex as described above. We then excite it by applying the laser potential with fixed axes $(X, Y=x, y$ in Eq. (10) for a short duration $t_{0}=0.5 \mathrm{~ms} \ll \omega_{\perp}^{-1}$. Thereby, the wave function $\psi_{0}$ of the condensate is transformed into

$$
\psi(\mathbf{r})=\psi_{0}(\mathbf{r})+\delta \psi(\mathbf{r}) \quad \text { with } \quad \delta \psi(\mathbf{r})=-\frac{i t_{0}}{2 \hbar} \epsilon M \omega_{\perp}^{2}\left(x^{2}-y^{2}\right) .
$$

This excites a superposition of $m=+2$ and $m=-2$ modes with equal amplitudes. More precisely, $\delta \psi$ can be written in terms of the usual $u_{ \pm}$and $v_{ \pm}$functions characterizing the quadrupole modes $m= \pm 2$ in a Bogoliubov analysis (see e.g. [26]) :

$$
\delta \psi(\mathbf{r})=i \epsilon^{\prime}\left(u_{+}(\mathbf{r})+v_{+}^{*}(\mathbf{r})\right)+i \epsilon^{\prime}\left(u_{-}(\mathbf{r})+v_{-}^{*}(\mathbf{r})\right)
$$

where $\epsilon^{\prime}$ is a dimensionless coefficient proportional to the strength $\epsilon$ and to the duration $t_{0}$ of the excitation.

We then let the cloud evolve freely in the magnetic trap for a variable time $t$. The condensate wave function becomes:

$$
\begin{aligned}
\psi(\mathbf{r}, t)=e^{-i \mu t / \hbar}\left\{\psi_{0}(\mathbf{r})\right. & +i \epsilon^{\prime} e^{-\gamma_{+} t}\left(e^{-i \omega_{+} t} u_{+}(\mathbf{r})+e^{i \omega_{+} t} v_{+}^{*}(\mathbf{r})\right) \\
& \left.+i \epsilon^{\prime} e^{-\gamma_{-} t}\left(e^{-i \omega_{-} t} u_{-}(\mathbf{r})+e^{i \omega_{-} t} v_{-}^{*}(\mathbf{r})\right)\right\},
\end{aligned}
$$

where $\mu$ is the chemical potential of the condensate. In (5) we added a phenomenological damping term for each mode. We then perform the time of flight analysis and measure the size $R_{1}$ and the polar angle $\theta$ of the long axis, the size $R_{\mathrm{s}}$ of the short axis, and 
calculate the cloud ellipticity $\zeta=R_{\mathrm{l}} / R_{\mathrm{s}}$. After some manipulation, we find that the predicted values for these quantities can be written in the following form:

$$
\cos 2 \theta=\frac{F(t)}{\sqrt{F^{2}(t)+G^{2}(t)}} \quad \sin 2 \theta=\frac{G(t)}{\sqrt{F^{2}(t)+G^{2}(t)}}
$$

and

$$
\zeta-1 \propto \sqrt{F^{2}(t)+G^{2}(t)},
$$

where the functions $F$ and $G$ are defined by

$$
\begin{aligned}
& F(t)=e^{-\gamma_{-} t} \sin \left(\omega_{-} t\right)+e^{-\gamma_{+} t} \sin \left(\omega_{+} t\right), \\
& G(t)=e^{-\gamma_{-} t} \cos \left(\omega_{-} t\right)-e^{-\gamma_{+} t} \cos \left(\omega_{+} t\right) .
\end{aligned}
$$

\subsection{Various time domains for the condensate precession}

We now discuss the behavior of the precessing condensate expected from Eqs. (66), assuming that the damping rates $\gamma_{ \pm}$of the quadrupole modes are much smaller than the frequencies $\omega_{ \pm}$.

3.2.1. Short time behavior. For times short compared to $\gamma_{ \pm}^{-1}$, the exponential decay of the functions $F$ and $G$ can be neglected and we obtain simply:

$$
\begin{aligned}
& \theta=\frac{\left(\omega_{+}-\omega_{-}\right) t}{4} \quad \text { modulo } \frac{\pi}{2}, \\
& \zeta-1 \propto\left|\sin \left(\left(\omega_{+}+\omega_{-}\right) t / 2\right)\right| .
\end{aligned}
$$

In this case, the lift of degeneracy $\omega_{+}-\omega_{-}$is simply obtained from the measurement of the precession angle $\theta$. The oscillation of the ellipticity is a function of the average frequency $\left(\omega_{+}+\omega_{-}\right) / 2$.

We note that combining (21) and (10), we obtain the result

$$
\theta=\frac{L_{z} t}{2 M r_{\perp}^{2}}
$$

For very short times (i.e. $\omega_{ \pm} t \ll 1$ ) this result is actually quite general, as we show below. It applies to any fluid confined in a harmonic potential with binary interactions, provided the fluid is initially in a stationary state with rotational invariance, described by a density operator $\rho_{0}$ :

$$
\begin{array}{lcc}
\left\langle x^{2}\right\rangle_{0}=\left\langle y^{2}\right\rangle_{0}=r_{\perp}^{2} / 2 & \langle x y\rangle_{0}=0 & \left\langle p_{x} p_{y}\right\rangle_{0}=0 \\
\left\langle x p_{x}+p_{x} x\right\rangle_{0}=\left\langle y p_{y}+p_{y} y\right\rangle_{0}=0 & \left\langle x p_{y}+y p_{x}\right\rangle_{0}=0
\end{array}
$$

Note that the system can have some angular momentum $\left(L_{z}=\left\langle x p_{y}-p_{y} x\right\rangle_{0} \neq 0\right)$. After the percussional excitation by the laser potential varying as $x^{2}-y^{2}$, the new density operator of the system is at first order in the perturbation:

$$
\rho=\rho_{0}-i \frac{\eta}{2}\left[\sum_{i=1}^{N} x_{i}^{2}-y_{i}^{2}, \rho_{0}\right],
$$


where the sum runs over the $N$ particles of the fluid. $x_{i}$ and $y_{i}$ denote the position operators of the $i$-th particle in the $x y$ plane. The real coefficient $\eta$ is a measure of the strength of the percussional excitation. We now calculate the time evolution of $\left\langle x^{2}\right\rangle_{t}$, $\left\langle y^{2}\right\rangle_{t}$ and $\langle x y\rangle_{t}$. Using Ehrenfest theorem we obtain:

$$
\frac{d}{d t}\left\langle x^{2}\right\rangle=\frac{i}{N \hbar} \sum_{i=1}^{N}\left\langle\left[H, x_{i}^{2}\right]\right\rangle=\frac{1}{m}\left\langle p_{x} x+x p_{x}\right\rangle .
$$

The average on the right hand side is calculated in the state (13) and we get for short times :

$$
\left\langle x^{2}\right\rangle_{t}=\frac{r_{\perp}^{2}}{2}-\frac{\hbar \eta t}{M} r_{\perp}^{2} \quad, \quad\left\langle y^{2}\right\rangle_{t}=\frac{r_{\perp}^{2}}{2}+\frac{\hbar \eta t}{M} r_{\perp}^{2}
$$

The calculation of $\langle x y\rangle$ is slightly more involved. It requires the value of $\left\langle x p_{y}+y p_{x}\right\rangle$ to first order in time and in $\eta$. We use the fact that in equilibrium $\left\langle x F_{y}+y F_{x}\right\rangle_{0}=0$, where $\mathbf{F}(\mathbf{r})$ is the force created on a given particle located in $\mathbf{r}$ by the $N-1$ remaining particles. We then obtain

$$
\left\langle x p_{y}+y p_{x}\right\rangle_{t}=-\frac{2 \eta \hbar L_{z} t}{M} \quad, \quad\langle x y\rangle_{t}=-\frac{\eta \hbar L_{z} t^{2}}{M^{2}} .
$$

Quite remarkably the results (14,[15) are independent of the binary interaction potential between the particles. The polar angle $\theta$ characterizing the long axis of the condensate is obtained from

$$
\tan 2 \theta=\frac{2\langle x y\rangle_{t}}{\left\langle x^{2}\right\rangle_{t}-\left\langle y^{2}\right\rangle_{t}}
$$

which is indeed equivalent to (12). The present proof, valid for any fluid confined in a harmonic potential with binary interactions, holds only for $t \ll \omega_{\perp}^{-1}$. As stated above, (10) holds also for much longer times $\left(\omega_{\perp}^{-1} \leq t \ll \gamma_{ \pm}^{-1}\right)$ for a pure BEC in the ThomasFermi limit [22].

3.2.2. Long time behavior. At longer times, the exponential decays of the amplitudes of the modes cannot be neglected anymore. If the two quadrupole modes decay with the same rate $\left(\gamma_{+}=\gamma_{-}\right)$, the result (10) is valid at any time. Simply the amplitude $\zeta$ of the quadrupole oscillation becomes weaker and weaker, which makes it harder to detect. On the contrary, if the two rates $\gamma_{+}$and $\gamma_{-}$are different, say e.g. $\gamma_{+}<\gamma_{-}$, one reaches at long time a regime where the mode $m=+2$ has an amplitude is much larger than $m=-2$. In this case one simply gets $\theta=\omega_{+} t / 2$ and $\zeta-1 \propto e^{-\gamma_{+} t}$.

\subsection{Experimental results}

Typical evolutions of the quantities $\theta$ and $\zeta$ are plotted in Fig. 4a. They have been obtained for a transverse frequency $\omega_{\perp} / 2 \pi=98.5 \mathrm{~Hz}$ and a nearly straight vortex $(\tau=2 \mathrm{~s})$. We observe the two successive regimes anticipated above. For short times, one observes the quadrupole oscillation with precessing axes. For $t>5 \mathrm{~ms}$, the precession rate increases and the jumps of $\pi / 2$ in $\theta$ become more and more rounded. This behavior 

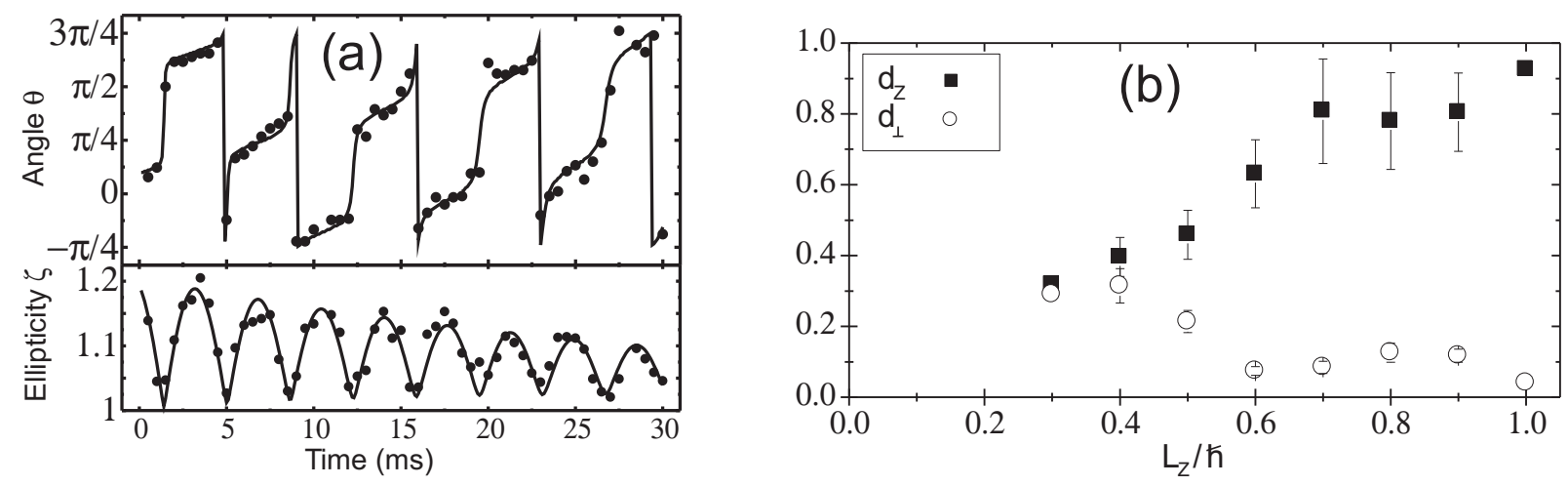

Figure 4. (a) Angle $\theta$ and ellipticity $\zeta$ as a function of time $t$ in presence of a single vortex. The value of the angular momentum per particle $L_{z}$ is deduced from the precession of the main axes observed for small $t$. (b) Variation of $d_{z}$ and $d_{\perp}$ as functions of $L_{z}$. All measurements were binned into intervals of $L_{z}=0.1 \hbar$ and averaged.

indicates that the mode $m=-2$ decays faster than $m=+2$, as discussed above. The fit using ([6)-(17) gives: $\omega_{+} / 2 \pi=159.5 \pm 1.0 \mathrm{~Hz}, \omega_{-} / 2 \pi=116.8 \pm 1.0 \mathrm{~Hz}, \gamma_{+}=19.1 \pm 2.0 \mathrm{~s}^{-1}$ and $\gamma_{-}=35.7 \pm 4.0 \mathrm{~s}^{-1}$. We note that the two measured frequencies satisfy the sum rule $\omega_{+}^{2}+\omega_{-}^{2}=4 \omega_{\perp}^{2}$ [22] with a good accuracy. From the difference $\omega_{+}-\omega_{-}$and the measured size of the condensate, we infer that the angular momentum per particle is $L_{z} \sim \hbar$ (to within 10\%) [27. The study of the difference between $\gamma_{+}$and $\gamma_{-}$is outside the scope of this paper and it will be discussed elsewhere [32.

\subsection{Relationship between $L_{z}$ and the shape of the vortex line}

If we are interested only in $L_{z}$, we do not have to repeat the full experimental sequence leading to Fig. Th. It is sufficient to perform only three experimental runs, which all start by preparing a condensate with a single vortex as described before. For the first two runs, we excite the two quadrupole surface modes $m= \pm 2$ and we probe the quadrupole oscillation at the two first maxima of the ellipticity $\zeta$ after the flash. From the precession of the condensate axes, we infer the frequency difference between the quadrupole modes, hence $L_{z}$ [22, 27]. For the third run, we repeat the experiment without the laser flash and analyze the shape of the vortex line. As above we extract the normalized length $d_{z}$ of the vortex line along the $z$ axis. We also measure the displacement $d_{\perp}$ of the axial part of the vortex line (bottom of the $\mathrm{U}$ ) from the center of the condensate, and normalize it by the radius of the condensate in the $x y$ plane (Fig. Ba a). Since we have access only to the projection of the decentering on the $x z$ plane, we actually measure $d_{\perp}|\cos \alpha|$, where $\alpha$ is the azimuthal angle of the axial part of the line. We account for this geometrical factor by dividing the measured displacement by $\langle|\cos \alpha|\rangle=2 / \pi$.

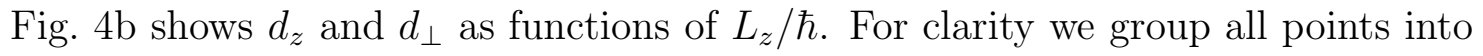
bins of $L_{z}=0.1 \hbar$ and average over $d_{z}$ and $d_{\perp}$. The error bars give the statistical variation. The data corresponding to $L_{z} \leq 0.2 \hbar$ are not reproducible enough and are omitted. A straight $\left(d_{z} \sim 1\right)$ and well centered $\left(d_{\perp} \ll 1\right)$ vortex line corresponds to 
$L_{z} \sim \hbar$. The graph shows that $d_{z} \simeq L_{z} / \hbar$. When $L_{z}$ decreases, we measure a decentering $d_{\perp} \leq 0.15$ as long as $L_{z}>0.5 \hbar$. Below $L_{z}=0.5 \hbar, d_{\perp}$ rises to 0.3 .

\subsection{Comparison with previous theoretical works}

We now compare our experimental results with recent predictions for the shape of a vortex line in an inhomogeneous cigar shaped condensate [11, 12, 13, 14, 15. These theoretical studies consist in looking for the ground state of the condensate in a frame rotating at an angular frequency $\Omega$. The general conclusion is that above a critical frequency $\Omega_{\mathrm{c}}$ the ground state of the system has one or several vortices. The central vortex is generally bent if the trap aspect ratio $\omega_{\perp} / \omega_{z}$ is large compared to 1 , which

is the case in our experiment. A precursor of this bending effect can also been found in [28, 29] in which a stability analysis of a straight vortex in an elongated condensate showed that some bending modes have negative energy, and are thus unstable.

Our experimental procedure is somewhat different from the one considered in these theoretical studies. $L_{z}$ and $\Omega$ play the role of a couple of conjugate extensive/intensive variables just like for instance volume and pressure. In our case no rotating anisotropy is imposed onto the condensate during the relevant evolution. The stirring laser has been switched on for a short time only at the beginning of the procedure in order to set a nonzero angular momentum in the system. We observe the evolution of the condensate in our static trap, as the angular momentum of the gas slowly decays. At first sight, neither $L_{z}$ seems to be conserved nor $\Omega$ imposed by the experimental environment. However, the long lifetimes observed for the vortex (up to $8 \mathrm{~s}$ ) suggest that during an interval of a fraction of a second (a typical thermalization time), $L_{z}$ is almost constant. Therefore the description of our rotating condensate at time $\tau$ should rather correspond to a system with a fixed $L_{z}$ than to a system rotating with a fixed $\Omega$. The states of minimal energy may differ between the two descriptions 14, 30. More precisely states with an angular momentum $L_{z}$ notably below $\hbar$ are found as saddle points of the energy functional calculated in the rotating frame (fixed $\Omega$ ), while they appear as energy minimum when one considers a condensate as ours, with a fixed angular momentum $L_{z}$ and where the $\Omega$ parameter is in principle not relevant 14 .

\section{Conclusion}

We have reported the complete observation of a single quantized vortex line. The Ushaped vortex line that we observe is remarkably similar to those predicted and plotted in [11, 12, 13, 14. As pointed out in [12, the bending of the vortex line is a symmetry breaking effect which does not depend on the presence of a rotating anisotropy and which happens even in a completely symmetric setup. We have given a detailed theoretical account of the relation between measured condensate deformations and the quadrupole mode characteristics (frequency and damping rate). The quadrupole oscillations of the rotating condensate were used to measure the angular momentum. The shape of the 
vortex line (bending and deviation from the center) were then related to the angular momentum of the system. Our results should help modelling the dissipative evolution of a rotating Bose-Einstein condensate.

We thank Y. Castin, F. Chevy, and G. Shlyapnikov for useful discussions. P. R. acknowledges support the Alexander-von-Humboldt Stiftung and by the EU (contract number HPMF CT 2000 00830). This work was partially supported by CNRS, Collège de France, Région Ile de France, DGA, DRED and EC (TMR network ERB FMRXCT96-0002).

[*] Unité de Recherche de l'Ecole normale supérieure et de l'Université Pierre et Marie Curie, associée au CNRS.

[1] R.J. Donnelly, Quantized Vortices in Helium II, (Cambridge, 1991).

[2] M.R. Matthews et al., Phys. Rev. Lett. 83, 2498 (1999).

[3] K.W. Madison et al., Phys. Rev. Lett. 84, 806, (2000).

[4] J.R. Abo-Shaeer et al., Science 292, 476 (2001); C. Raman et al., Phys. Rev. Lett. 87, 210402 (2001).

[5] E. Hodby et al., Phys. Rev. Lett. 86, 2196 (2001).

[6] P.C. Haljan et al., Phys. Rev. Lett. 87, 210403 (2001).

[7] M. Tinkham, Introduction to superconductivity (McGraw-Hill, 1996).

[8] A. Tonomura et al., Nature 412, 620 (2001); A. Tonomura et al., Phys. Rev. Lett. 88, 237001 (2002).

[9] P. Engels et al., Phys. Rev. Lett. 89, 100403 (2002).

[10] P. Rosenbusch, V. Bretin, and J. Dalibard, Phys. Rev. Lett. 89, 200403 (2002).

[11] J.J. García-Ripoll and V.M. Pérez-García, Phys. Rev. A 63, 041603 (2001).

[12] J.J. García-Ripoll and V.M. Pérez-García, Phys. Rev. A 64, 053611 (2001).

[13] A. Aftalion and T. Riviere, Phys. Rev. A 64, 043611 (2001).

[14] M. Modugno et al., Eur. Phys. J. D 22, 235 (2003).

[15] A. Aftalion and R. L. Jerrard, cond-mat/0204475.

[16] K.W. Madison et al., Phys. Rev. Lett. 86, 4443 (2001).

[17] Y. Castin and R. Dum, Phys. Rev. Lett. 77, 5315 (1996).

[18] J.R. Abo-Shaeer et al., Phys. Rev. Lett. 88, 070409 (2002).

[19] O.N. Zhuravlev et al., Phys. Rev. A 64, 053601 (2001).

[20] D. Guéry-Odelin, Phys. Rev. A 62, 033607 (2000).

[21] P.O. Fedichev and G.V. Shlyapnikov, Phys. Rev. A 60, R1779 (1999).

[22] F. Zambelli and S. Stringari, Phys. Rev. Lett. 81, 1754 (1998).

[23] R. Dodd et al., Phys. Rev. A 56, 587 (1997).

[24] S. Sinha, Phys. Rev. A 55, 4325 (1997).

[25] A. Svidzinsky and A. Fetter, Phys. Rev. A. 58, 3168 (1998).

[26] P. Ohberg et al., Phys. Rev. A 56, R3346 (1997).

[27] F. Chevy et al., Phys. Rev. Lett. 85, 2223 (2000).

[28] A.A. Svidzinsky and A.L. Fetter, Phys. Rev. A 62, 063617 (2000).

[29] D.L. Feder et al., Phys. Rev. Lett. 86, 564 (2001).

[30] D.A. Butts and D. S. Rokshar, Nature 397, 327 (1999).

[31] S. Stringari, Phys. Rev. Lett. 77, 2360-2363 (1996)

[32] V. Bretin, P. Rosenbusch, F. Chevy, G.V. Shlyapnikov, and J. Dalibard, to be published. 